\title{
How dynamic docking simulations can help to tackle tough drug targets
}

\author{
Maurizio Recanatini*,1 \\ ${ }^{1}$ Department of Pharmacy \& Biotechnology, University of Bologna, Via Belmeloro 6, 40126 Bologna, Italy \\ *Author for correspondence: maurizio.recanatini@unibo.it \\ “dynamic docking is the simultaneous exploration of conformations and binding modes by means \\ of classical MD simulations."
}

First draft submitted: 4 July 2018; Accepted for publication: 30 October 2018; Published online: 10 December 2018

Keywords: computational chemistry and molecular modeling • drug discovery • molecular dynamics

The docking simulation is one of the computational tools routinely used by all drug design groups either to investigate a ligand's binding mode or to virtually screen a library of putative hits. Since the beginning of the docking era, it has been realized that one of the crucial issues to be tackled was the flexibility of both molecules involved in the complex, that is, the target macromolecule (usually a protein) and the ligand. In their 1982 seminal paper on docking [1], Kuntz et al. stated, "without allowing molecular flexibility, many aspects of ligand-receptor interactions are not properly described." Nowadays, practically all docking programs include the possibility to account for the conformational flexibility of the ligand, and some of them allow considering partially the target flexibility (in the 'easiest' case, the amino acid side chains). However, even with these kinds of adjustments, some important aspects of the ligand-protein complex formation are completely neglected, to name one, the loop rearrangements that can occur in the case of highly flexible binding sites (in other words, the induced-fit phenomenon). To overcome these limitations, molecular dynamics (MD) simulations have been introduced in the practice of docking, such that a combined use of docking and MD started to catch on gradually, and the sequential application of the two approaches led to relevant improvements in the binding mode identification. The generation of an ensemble of protein conformations, on which to carry out the virtual docking experiments, as well as the validation and refinement of docking poses through MD simulation runs are examples of this combination strategy [2].

In the last decade, also thanks to the dramatic improvements in both computing power and algorithm efficiency, the scale of the biological phenomena amenable to MD simulations has increased substantially. In other words, we are now able to simulate processes like the association of ligand and protein in solution from the very beginning, that is, starting from a system configuration where the two species are several Angstrom apart. This is what we call dynamic docking that differs conceptually and technically from standard docking in the same way as a full movie differs from the last frame. Actually, the term 'dynamic docking' was used first by Hoffmann to name what he called a 'new paradigm for protein-protein reactions' [3], but it was recently proposed to indicate an evolution of the classical docking concept referred to a small molecule binding to a macromolecule [2,4]. In the essence, what characterizes dynamic docking is the simultaneous exploration of conformations and binding modes by means of classical MD simulations. This approach takes along several pros and cons; among the former, the possibility to calculate macroscopic properties of the system through statistical mechanics [5], and to obtain a detailed description of the association path (including the role of water molecules); and among the latter, the computational and time resources required for (repeated) MD simulations on large systems that are rather high and not compatible yet with the needs of industrial drug discovery.

In an effort to exploit to the best the opportunities offered by dynamic docking simulations while minimizing the computational costs, several groups have proposed and tested MD-based methods, which permit to increase the speed and efficiency of configuration sampling and to calculate the observables inherent to the binding process (two aspects, which are parallel, in some ways, the pose generation and scoring of standard docking). As regards 
the sampling, so-called 'enhanced sampling methods' are currently available at affordable computational costs. They aim at exploring the energy landscape of the system as thoroughly as possible, that is, trying both to avoid being trapped in energy minima, and, crucial for dynamic docking simulations, to accelerate the sampling of 'rare events' (like binding and unbinding). As regards the calculation of observables, in this context it refers mainly to the estimation of the binding-free energy $\left(\Delta \mathrm{G}_{\mathrm{b}}\right)$ from the MD trajectories through statistical mechanics. $\Delta \mathrm{G}_{\mathrm{b}}$ is not easy to assess accurately, and the just mentioned configuration sampling procedure is critical in conferring statistical robustness to the evaluation procedure. Recently, adaptive sampling methods relying on the Markov state models framework [6] have been successfully introduced in this field. The reader who is interested in the recent advancements of MD simulations with implications in dynamic docking can refer to the review of De Vivo et al. [7].

The first example of dynamic docking simulation of pharmacologically relevant ligand-target complexes came from the Parrinello group, who in 2005 applied the metadynamics method to reproduce experimental binding poses and $\Delta G_{b}$ values of four ligand-receptor systems [8]. Since then, a growing number of reports are appearing in the literature (for a recent review see [2]), where the emphasis is often cast on the sampling method or the data treatment approach, while the target proteins in most cases are conventional and well known. However, it might be of interest to start challenging the limits of applicability of this approach, by checking the performance of the method on less conventional, putatively difficult ('tough') targets. For the purpose of this Editorial, the author proposes to consider difficult targets, flat protein surfaces (typically, but not only, protein-protein interfaces); cryptic binding sites; and, intrinsically disordered proteins, all of which, by the way, have raised pharmacological interest.

With regard to the flat protein surfaces, this kind of target has to be dealt with when searching for proteinprotein interaction disruptors. The difficulty one encounters when attempting structure-based ligand design for such targets arises from the fact that the standard docking algorithms are tailored for treating binding 'cavities' or 'pockets', that are quite different in shape and area from protein-protein interfaces. It may not be coincidental that some clean examples of dynamic docking simulations in this field come from the reproduction of the binding mode of peptides to proteins. Both Cuzzolin et al. [9] and Spitaleri et al. [10] examined well-characterized peptide complexes where protein-derived peptides bind their target protein domain: $\mathrm{BclX}_{\mathrm{L}}-\mathrm{BAD}$ and $\mathrm{MDM} 2-\mathrm{p} 53$, and RAD51-BRCA2, respectively. In the first case, the authors employed a supervised adaptive MD method where the supervision helped reducing the simulation time, while in the second case an MD-based method was used that took advantage of a biasing electrostatic potential acting on the heavy atoms of both the ligand and some binding site residues. Interestingly, in a context different from protein-protein interactions, Yuan et al. reported a simulation of the binding of a ligand, BPTU, to the extra-helical site of a GPCR, the P2Y $\mathrm{Y}_{1}$ receptor [11]. The crystallographically determined binding site of BPTU is external to the helix bundle and membrane exposed: not a classical binding pocket indeed. The authors were able to define the ligand-protein association pathway across the membrane lipid bilayer, as well as to reconstruct the free energy surface and calculate the $\Delta \mathrm{G}_{\mathrm{b}}$ through the combined use of conventional MD and enhanced sampling methods.

Cryptic sites can be considered as those sites that manifest themselves only upon ligand binding and that are not evident from the 3D structure of the protein obtained either from biophysical experiments or through modeling. In other words, they cannot be identified on the free energy surface of the (apo)protein. Therefore, in principle, should a drug candidate bind to a cryptic pocket of a receptor, it could not be identified by a virtual screening campaign based on a standard docking procedure. In these cases, dynamic docking simulations might be the right tool to reveal the binding mechanism. A brilliant example thereof has been reported by the De Fabritiis group, who succeeded in identifying a cryptic binding site for an antagonist of the D3 dopamine receptor [12]. To obtain this result, Ferruz et al. performed high-throughput unbiased MD simulations (reaching a total aggregated simulation time of about $700 \mu \mathrm{s}$ ) and used Markov state model to analyze the trajectories and reconstruct the binding pathway of the ligand. They contrasted the outcome of the dynamic study with standard docking results showing that only the former approach was able to provide a ligand binding mechanism consistent with single point mutation experiments.

Proteins that lack a stable secondary or tertiary structure are classified as intrinsically disordered (IDPs), and there is evidence that a growing number of them are valuable targets for drug discovery [13]. In the literature, some efforts are reported aimed at simulating the interaction of small molecules and IDPs eventually in view of the possible development of a virtual screening protocol able to prioritize IDP ligands. However, the standard docking approach is applied even though taking advantage of MD simulations. As an example, the work of a group from Peking University showed how to exploit both the ensemble docking and the MD-based pose refinement strategies to study and identify ligands able to bind to the disordered domain of the oncotarget c-Myc [14,15]. Indeed, because of their 
rather flat energy landscape, IDPs pose a formidable challenge for any simulation attempt aimed at investigating the ligand-target binding mechanism. Actually, the Liu's picture of 'ligand clouds around protein clouds' [14] is quite pertinent and calls for some new idea to tackle the problem. Might dynamic docking be the answer? Clear examples thereof have not appeared yet, even though the Vendruscolo's metadynamic metainference approach [16] seems to provide a significant step forward toward the simulation of such fuzzy systems as the ligand-IDP complexes. Actually, by combining experimental biophysical techniques and these NMR-restrained simulations, Heller et al. were able to determine what they called the 'diffuse-specific' binding mechanism of a small molecule ligand to an intrinsically disordered region of c-Myc [17].

In conclusion, dynamic docking is likely to acquire a central role as regards the simulation of ligand-target systems, even when the latter counterpart is 'tough'. Taking the examples above, despite their scarcity, one can see that the plasticity of the protein plays a key role in the process of binding, possibly more than in the case of conventional pockets. Considering this can be crucial, and MD tools, on which dynamic docking is based, are the best suited to account for such plasticity. Problems are still present (first of all the computational costs for exhaustive sampling), challenges are open (mainly IDPs), but opportunities are quite exciting (and the article has not mentioned the possibility offered by these kind of simulations of accessing the kinetics of the system, but that is another story).

\section{Financial \& competing interests disclosure}

The authors have no relevant affiliations or financial involvement with any organization or entity with a financial interest in or financial conflict with the subject matter or materials discussed in the manuscript. This includes employment, consultancies, honoraria, stock ownership or options, expert testimony, grants or patents received or pending, or royalties.

No writing assistance was utilized in the production of this manuscript.

\section{References}

1. Kuntz ID, Blaney JM, Oatley SJ et al. A geometric approach to macromolecule-ligand interactions. J. Mol. Biol. 161(2), 269-288 (1982).

2. Gioia D, Bertazzo M, Recanatini M et al. Dynamic docking: a paradigm shift in computational drug discovery. Molecules $22(11)$, 2029 (2017).

3. Liang Z-X, Nocek JM, Huang K et al. Dynamic docking and electron transfer between Zn-myoglobin and cytochrome b5. J. Am. Chem. Soc. 124(24), 6849-6859 (2002).

4. De Vivo M, Cavalli A. Recent advances in dynamic docking for drug discovery. WIREs Comput. Mol. Sci. 7(6), e1320 (2017).

5. Coveney PV, Wan S. On the calculation of equilibrium thermodynamic properties from molecular dynamics. Phys. Chem. Chem. Phys. 18(44), 30236-30240 (2016).

6. Husic BE, Pande VS. Markov state models: from an art to a science. J. Am. Chem. Soc. 140(7), 2386-2396 (2018).

7. De Vivo M, Masetti M, Bottegoni G, Cavalli A. Role of molecular dynamics and related methods in drug discovery. J. Med. Chem. 59(9), 4035-4061 (2016).

8. Gervasio FL, Laio A, Parrinello M. Flexible docking in solution using metadynamics. J. Am. Chem. Soc. 127(8), 2600-2607 (2005).

9. Salmaso V, Sturlese M, Cuzzolin A, Moro S. Exploring protein-peptide recognition pathways using a supervised molecular dynamics approach. Structure 25(4), 655-662.e2 (2017).

10. Spitaleri A, Decherchi S, Cavalli A, Rocchia W. Fast dynamic docking guided by adaptive electrostatic bias: the MD-binding approach. J. Chem. Theory Comput. 14(3), 1727-1736 (2018).

11. Yuan X, Raniolo S, Limongelli V, Xu Y. The molecular mechanism underlying ligand binding to the membrane-embedded site of a G-protein-coupled receptor. J. Chem. Theory Comput. 14(5), 2761-2770 (2018).

12. Ferruz N, Doerr S, Vanase-Frawley MA et al. Dopamine D3 receptor antagonist reveals a cryptic pocket in aminergic GPCRs. Sci. Rep. 8(1), 897 (2018).

13. Uversky VN, Davé V, Iakoucheva LM et al. Pathological unfoldomics of uncontrolled chaos: intrinsically disordered proteins and human diseases. Chem. Rev. 114(13), 6844-6879 (2014).

14. Jin F, Yu C, Lai L, Liu Z. Ligand clouds around protein clouds: a scenario of ligand binding with intrinsically disordered proteins. PLoS Comput. Biol. 9(10), e1003249 (2013).

15. Yu C, Niu X, Jin F et al. Structure-based inhibitor design for the intrinsically disordered protein c-Myc. Sci. Rep. 6, 22298 (2016).

16. Bonomi M, Camilloni C, Vendruscolo M. Metadynamic metainference: enhanced sampling of the metainference ensemble using metadynamics. Sci. Rep. 6, 31232 (2016).

17. Heller GT, Aprile FA, Bonomi M et al. Sequence specificity in the entropy-driven binding of a small molecule and a disordered peptide. J. Mol. Biol. 429(18), 2772-2779 (2017). 